\title{
HOCHSCHILD COHOMOLOGY RING OF A MAXIMAL ORDER OF THE QUATERNION ALGEBRA
}

\author{
By
}

\author{
Takao HaYami
}

\begin{abstract}
We give an efficient bimodule projective resolution of a maximal $\mathbf{Z}$-order $\Lambda$ of the ordinary quaternion algebra over $\mathbf{Q}$, and therefore we determine the ring structure of the Hochschild cohomology of $\Lambda$ by calculating the Yoneda products using this resolution.
\end{abstract}

\section{Introduction}

Let $R$ be a commutative ring and $\Lambda$ an $R$-algebra which is a finitely generated projective $R$-module. The $n$th Hochschild cohomology of $\Lambda$ is defined by $H H^{n}(\Lambda):=\operatorname{Ext}_{\Lambda^{\mathrm{e}}}^{n}(\Lambda, \Lambda)$, where $\Lambda^{\mathrm{e}}=\Lambda \otimes_{R} \Lambda^{\mathrm{op}}$. The Yoneda product gives $H H^{*}(\Lambda):=\bigoplus_{n \geq 0} H H^{n}(\Lambda)$ a graded ring structure with $1 \in Z \Lambda \simeq H H^{0}(\Lambda)$ where $Z \Lambda$ denotes the center of $\Lambda . H H^{*}(\Lambda)$ is called the Hochschild cohomology ring of $\Lambda$. The Hochschild cohomology ring $H H^{*}(\Lambda)$ is graded-commutative, that is, for $\alpha \in H H^{p}(\Lambda)$ and $\beta \in H H^{q}(\Lambda)$ we have $\alpha \beta=(-1)^{p q} \beta \alpha$ (see [2], [6]).

The Hochschild cohomology has important connections and applications to the representation theory of algebras. For example, under appropriate hypotheses two derived equivalent algebras have isomorphic Hochschild cohomology algebras (see [5, Proposition 2.5]), and the second Hochschild cohomology group is important in deformation theory. The Hochschild cohomology has been studied for various algebras, however it is difficult to compute in general.

We have investigated the Hochschild cohomology of quaternion orders in [3] (see also [6], [7], [4]). Let $A=\mathbf{Q} \oplus \mathbf{Q} i \oplus \mathbf{Q} j \oplus \mathbf{Q} i j$ be the ordinary quaternion algebra over $\mathbf{Q}$ with the relations $i^{2}=j^{2}=-1$, $i j=-j i$. We set $\Gamma=\mathbf{Z} \oplus \mathbf{Z} i \oplus$

2000 Subject Classification: 16E40.

Key Words: Hochschild cohomology ring, bimodule projective resolution, maximal quaternion order, Yoneda product.

Received June 20, 2011. 
$\mathbf{Z}_{j} \oplus \mathbf{Z} i j$. Then $\Gamma$ is a $\mathbf{Z}$-order of $A$. The multiplicative structure of the Hochschild cohomology ring $H H^{*}(\Gamma)$ is known in [6] (see [3] for a new proof and a generalization):

$$
H H^{*}(\Gamma) \simeq \mathbf{Z}[X, Y, Z] /\left(2 X, 2 Y, 2 Z, X^{2}+Y^{2}+Z^{2}\right),
$$

where $\operatorname{deg} X=\operatorname{deg} Y=\operatorname{deg} Z=1$. So it is natural ask to consider the Hochschild cohomology rings of other quaternion orders. On the other hand, Bobovich [1] shows that the Hochschild cohomology of a maximal order of a simple central algebra over the algebraic number field is periodic with period 2 . Now we set $a=(1+i+j+i j) / 2$. Then $\Lambda=\mathbf{Z} \oplus \mathbf{Z} i \oplus \mathbf{Z} j \oplus \mathbf{Z} a$ is a maximal $\mathbf{Z}$-order of $A$. In this article, we give an explicit bimodule projective resolution of a maximal quaternion order $\Lambda$, and apply the result to determine the Hochschild cohomology ring of $\Lambda$. This resolution is not periodic, but nevertheless we can determine the ring structure of the Hochschild cohomology by using this resolution.

In Section 2, we give a bimodule projective resolution of $\Lambda$ (Theorem 2.1). In Section 3, we give the module structure of $H H^{*}(\Lambda)$ using this resolution of $\Lambda$ (Theorem 3.1). This is a complicated calculation. To compute the Yoneda products on $H H^{*}(\Lambda)$ we need generators of $H H^{*}(\Lambda)$ as a module. In Section 4, as a main theorem of this paper, we determine the Hochschild cohomology ring $H H^{*}(\Lambda)$ (Theorem 4.2).

\section{Bimodule Projective Resolution}

Let $A=\mathbf{Q} \oplus \mathbf{Q} i \oplus \mathbf{Q} j \oplus \mathbf{Q} i j$ be the ordinary quaternion algebra over $\mathbf{Q}$ with the relations $i^{2}=j^{2}=-1, i j=-j i$. We put $a=(1+i+j+i j) / 2$. Then $\Lambda=$ $\mathbf{Z} \oplus \mathbf{Z} i \oplus \mathbf{Z} j \oplus \mathbf{Z} a$ is a maximal order of $A$. Note that the following equations hold:

$$
i a=a-1-j=i-1-a i, \quad j a+a j=j-1, \quad a^{2}=a-1, \quad a^{3}=-1 .
$$

It is easy to see that $1, i, a$, ia are linearly independent over $\mathbf{Z}$, so we take $\{1, i, a, i a\}$ as a Z-basis of $\Lambda$.

In this section we give an efficient bimodule projective resolution of $\Lambda$. For each $q \geq 0$, let $Y_{q}$ be the direct sum of $q+1$ copies of $\Lambda \otimes \Lambda$. As elements of $Y_{q}$, we set

$$
c_{q}^{s}= \begin{cases}(0, \ldots, 0,1 \stackrel{s}{\otimes} 1,0, \ldots, 0) & (\text { if } 1 \leq s \leq q+1), \\ 0 & \text { (otherwise) } .\end{cases}
$$


Then we have $Y_{q}=\bigoplus_{k=1}^{q+1} \Lambda c_{q}^{k} \Lambda$. Define left $\Lambda^{\mathrm{e}}$-homomorphisms $\pi: Y_{0} \rightarrow \Lambda$; $c_{0}^{1} \mapsto 1$ and $\delta_{q}: Y_{q} \rightarrow Y_{q-1}(q>0)$ given by

$$
\delta_{q}\left(c_{q}^{s}\right)= \begin{cases}i c_{q-1}^{s}+c_{q-1}^{s} i+a c_{q-1}^{s-1}+c_{q-1}^{s-1} a-c_{q-1}^{s-1} & \text { for } q \text { even } \\ i c_{q-1}^{s}-c_{q-1}^{s} i+a c_{q-1}^{s-1}-c_{q-1}^{s-1} a & \text { for } q \text { odd }\end{cases}
$$

THEOREM 2.1. The above $(Y, \pi, \delta)$ is a $\Lambda^{\mathrm{e}}$-projective resolution of $\Lambda$.

Proof. First, we must check that $\pi \cdot \delta_{1}=0$ and $\delta_{q} \cdot \delta_{q+1}=0$ for $q \geq 1$. Clearly $\pi \cdot \delta_{1}=0$ holds. If $q(\geq 2)$ is even, we have the following:

$$
\begin{aligned}
\delta_{q-1} \cdot \delta_{q}\left(c_{q}^{s}\right)= & \delta_{q-1}\left(i c_{q-1}^{s}+c_{q-1}^{s} i+a c_{q-1}^{s-1}+c_{q-1}^{s-1} a-c_{q-1}^{s-1}\right) \\
= & i\left(i c_{q-2}^{s}-c_{q-2}^{s} i\right)+\left(i c_{q-2}^{s}-c_{q-2}^{s} i\right) i+i\left(a c_{q-2}^{s-1}-c_{q-2}^{s-1} a\right) \\
& +\left(a c_{q-2}^{s-1}-c_{q-2}^{s-1} a\right) i+a\left(i c_{q-2}^{s-1}-c_{q-2}^{s-1} i\right)+\left(i c_{q-2}^{s-1}-c_{q-2}^{s-1} i\right) a \\
& -\left(i c_{q-2}^{s-1}-c_{q-2}^{s-1} i\right)+a\left(a c_{q-2}^{s-2}-c_{q-2}^{s-2} a\right)+\left(a c_{q-2}^{s-2}-c_{q-2}^{s-2} a\right) a \\
& -\left(a c_{q-2}^{s-2}-c_{q-2}^{s-2} a\right) \\
= & (i a+a i-i) c_{q-2}^{s-1}-c_{q-2}^{s-1}(a i+i a-i)+\left(a^{2}-a\right) c_{q-2}^{s-2}-c_{q-2}^{s-2}\left(a^{2}-a\right) \\
= & 0 .
\end{aligned}
$$

The case $q$ odd is similar.

Next we state a contracting homotopy. We define right $\Lambda$-homomorphisms $T_{-1}: \Lambda \rightarrow Y_{0}$ and $T_{q}: Y_{q} \rightarrow Y_{q+1}(q \geq 0)$ as follows:

$$
\begin{aligned}
T_{-1}(\lambda) & =c_{0}^{1} \lambda \quad(\text { for } \lambda \in \Lambda) ; \\
T_{q}\left(i^{m} c_{q}^{s}\right) & = \begin{cases}m c_{q+1}^{1} & (s=1), \\
0 & (s \geq 2),\end{cases} \\
T_{q}\left(i^{m} a c_{q}^{s}\right) & = \begin{cases}m c_{q+1}^{1}-m c_{q+1}^{1} a+i^{m} c_{q+1}^{2} & (s=1 \text { and } q \text { odd }), \\
m c_{q+1}^{1} a+i^{m} c_{q+1}^{2} & (s=1 \text { and } q \text { even }), \\
i^{m} c_{q+1}^{s+1} & (s \geq 2),\end{cases}
\end{aligned}
$$

where $m=0,1$. These homomorphisms are right $\Lambda$-homomorphism. So it permits us to cut down the number of cases. Thus we must check that

$$
\left(\delta_{q+1} T_{q}+T_{q-1} \delta_{q}\right)\left(i^{m} a^{n} c_{q}^{s}\right)=i^{m} a^{n} c_{q}^{s}
$$


holds for $m=0,1 ; n=0,1 ; 1 \leq s \leq q+1$, where we set $\delta_{0}=\pi$. In the case $q=0$, we have the following:

$$
\delta_{1} T_{0}\left(i^{m} a^{n} c_{0}^{1}\right)+T_{-1} \pi\left(i^{m} a^{n} c_{0}^{1}\right)=\left(i^{m} a^{n} c_{0}^{1}-c_{0}^{1} i^{m} a^{n}\right)+c_{0}^{1} i^{m} a^{n}=i^{m} a^{n} c_{0}^{1}
$$

Note that the computation of $\delta_{1} T_{0}\left(i^{m} a^{n} c_{0}^{1}\right)$ is divided into four cases.

In the case $q$ odd, we have the following:

Case $s=1$ :

$$
\begin{aligned}
\delta_{q+1} T_{q}\left(i^{m} c_{q}^{1}\right) & =\delta_{q+1}\left(m c_{q+1}^{1}\right)=m\left(i c_{q}^{1}+c_{q}^{1} i\right) \quad(m=0,1), \\
T_{q-1} \delta_{q}\left(i^{m} c_{q}^{1}\right) & =T_{q-1}\left(i^{m}\left(i c_{q-1}^{1}-c_{q-1}^{1} i\right)\right)= \begin{cases}c_{q}^{1} & (m=0), \\
-c_{q}^{1} i & (m=1),\end{cases} \\
\delta_{q+1} T_{q}\left(i^{m} a c_{q}^{1}\right) & =\delta_{q+1}\left(m c_{q+1}^{1}-m c_{q+1}^{1} a+i^{m} c_{q+1}^{2}\right)
\end{aligned}
$$

Case $s \geq 2$ :

$$
\begin{aligned}
\delta_{q+1} T_{q}\left(i^{m} c_{q}^{s}\right) & =0 \\
T_{q-1} \delta_{q}\left(i^{m} c_{q}^{s}\right) & =T_{q-1}\left(i^{m}\left(i c_{q-1}^{s}-c_{q-1}^{s} i+a c_{q-1}^{s-1}-c_{q-1}^{s-1} a\right)\right)=i^{m} c_{q}^{s}, \\
\delta_{q+1} T_{q}\left(i^{m} a c_{q}^{s}\right) & =\delta_{q+1}\left(i^{m} c_{q+1}^{s+1}\right) \\
& =i^{m}\left(i c_{q}^{s+1}+c_{q}^{s+1} i+a c_{q}^{s}+c_{q}^{s} a-c_{q}^{s}\right), \\
T_{q-1} \delta_{q}\left(i^{m} a c_{q}^{s}\right) & =T_{q-1}\left(i^{m}\left((i-1-i a) c_{q-1}^{s}-a c_{q-1}^{s} i+(a-1) c_{q-1}^{s-1}-a c_{q-1}^{s-1} a\right)\right) \\
& =-i^{m}\left(i c_{q}^{s+1}+c_{q}^{s+1} i+c_{q}^{s} a-c_{q}^{s}\right) .
\end{aligned}
$$


Note that the computations of $T_{q-1} \delta_{q}$ are divided into some subcases. The case $q(\geq 2)$ even is handled by a similar way.

It seems to be difficult (or impossible) to construct a periodic bimodule projective resolution of $\Lambda$. The resolution in Theorem 2.1 is not periodic, but nevertheless we can determine the ring structure of the Hochschild cohomology by using this resolution.

\section{Module Structure}

In this section, we determine the module structure of $H H^{n}(\Lambda)$. To calculate products on $H H^{*}(\Lambda)$ by the Yoneda product, we need an explicit module generator of $H H^{n}(\Lambda)$. Let $\Lambda^{q+1}$ be a direct sum of $q+1$ copies of $\Lambda$. As elements of $\Lambda^{q+1}$, we set

$$
l_{q}^{s}= \begin{cases}(0, \ldots, 0, \stackrel{s}{1}, 0, \ldots, 0) & (\text { if } 1 \leq s \leq q+1), \\ 0 & \text { (otherwise) } .\end{cases}
$$

Thus we have $\Lambda^{q+1}=\bigoplus_{k=1}^{q+1} \Lambda l_{q}^{k}$.

Applying the functor $\operatorname{Hom}_{\Lambda^{\mathrm{e}}}(-, \Lambda)$ to the resolution $(Y, \pi, \delta)$, we have the following complex, where we identify $\operatorname{Hom}_{\Lambda^{\mathrm{e}}}\left(Y_{q}, \Lambda\right)$ with $\Lambda^{q+1}$ using an isomorphism $\operatorname{Hom}_{\Lambda^{\mathrm{e}}}\left(Y_{q}, \Lambda\right) \rightarrow \Lambda^{q+1} ; f \mapsto \sum_{k=1}^{q+1} f\left(c_{q}^{k}\right) l_{q}^{k}$ :

$$
\begin{gathered}
\left(\operatorname{Hom}_{\Lambda^{\mathrm{e}}}(Y, \Lambda), \delta^{\#}\right): \quad 0 \longrightarrow \Lambda \stackrel{\delta_{1}^{\#}}{\longrightarrow} \Lambda^{2} \stackrel{\delta_{2}^{\#}}{\longrightarrow} \Lambda^{3} \stackrel{\delta_{3}^{\#}}{\longrightarrow} \Lambda^{4} \stackrel{\delta_{4}^{\#}}{\longrightarrow} \Lambda^{5} \longrightarrow \cdots, \\
\delta_{q+1}^{\#}\left(\lambda l_{q}^{s}\right)= \begin{cases}i \lambda l_{q+1}^{s}-\lambda i l_{q+1}^{s}+a \lambda l_{q+1}^{s+1}-\lambda a l_{q+1}^{s+1} & \text { for } q \text { odd }, \\
i \lambda l_{q+1}^{s}+\lambda i l_{q+1}^{s}+a \lambda l_{q+1}^{s+1}+\lambda a l_{q+1}^{s+1}-\lambda l_{q+1}^{s+1} & \text { for } q \text { even },\end{cases}
\end{gathered}
$$

for $\lambda \in \Lambda$. In the above, note that

$$
\lambda l_{q}^{s}= \begin{cases}(0, \ldots, 0, \stackrel{s}{\lambda}, 0, \ldots, 0) & (\text { if } 1 \leq s \leq q+1), \\ 0 & \text { (otherwise) }\end{cases}
$$

and so on. In the following, if $z$ is a cocycle, we also denote its cohomology class by $z$ for brevity.

THEOREM 3.1. The module structure of $H H^{n}(\Lambda)$ is as follows:

$$
H H^{n}(\Lambda)= \begin{cases}\mathbf{Z} & (n=0), \\ 0 & (n \text { odd }), \\ (\mathbf{Z} / 2 \mathbf{Z}) l_{n}^{1} & (n(\neq 0) \text { even }) .\end{cases}
$$


Proof. Let $\lambda_{k}=b_{k}+c_{k} i+d_{k} a+e_{k} i a\left(b_{k}, c_{k}, d_{k}, e_{k} \in \mathbf{Z}\right)$ be any element of $\Lambda$. Then we have

$$
\begin{aligned}
i \lambda_{k} & =b_{k} i-c_{k}+d_{k} i a-e_{k} a, \\
\lambda_{k} i & =b_{k} i-c_{k}+d_{k}(i-1-i a)+e_{k}(a-1-i) \\
& =-\left(c_{k}+d_{k}+e_{k}\right)+\left(b_{k}+d_{k}-e_{k}\right) i+e_{k} a-d_{k} i a, \\
a \lambda_{k} & =b_{k} a+c_{k}(i-1-i a)+d_{k}(a-1)+e_{k}(i-a) \\
& =-\left(c_{k}+d_{k}\right)+\left(c_{k}+e_{k}\right) i+\left(b_{k}+d_{k}-e_{k}\right) a-c_{k} i a, \\
\lambda_{k} a & =b_{k} a+c_{k} i a+d_{k}(a-1)+e_{k} i(a-1) \\
& =-d_{k}-e_{k} i+\left(b_{k}+d_{k}\right) a+\left(c_{k}+e_{k}\right) i a .
\end{aligned}
$$

(i) The case $n=0$ : We see that $H H^{0}(\Lambda)=\operatorname{Ker} \delta_{1}^{\#}=\mathbf{Z}$. In fact, for $\lambda_{1}=$ $b_{1}+c_{1} i+d_{1} a+e_{1} i a\left(b_{1}, c_{1}, d_{1}, e_{1} \in \mathbf{Z}\right)$, we have

$$
\begin{aligned}
\lambda_{1} \in \operatorname{Ker} \delta_{1}^{\#} & \Leftrightarrow\left\{\begin{array}{l}
i \lambda_{1}-\lambda_{1} i=0, \\
a \lambda_{1}-\lambda_{1} a=0
\end{array}\right. \\
& \Leftrightarrow\left\{\begin{array}{l}
\left(d_{1}+e_{1}\right)+\left(e_{1}-d_{1}\right) i-2 e_{1} a+2 d_{1} i a=0, \\
-c_{1}+\left(c_{1}+2 e_{1}\right) i-e_{1} a-\left(2 c_{1}+e_{1}\right) i a=0
\end{array}\right. \\
& \Leftrightarrow c_{1}=d_{1}=e_{1}=0 .
\end{aligned}
$$

(ii) The case $n$ odd: We show $H H^{n}(\Lambda)=0$. Since the cohomology module is periodic with period 2, it suffices to show $H H^{1}(\Lambda)=0$. For $\lambda_{k}=b_{k}+c_{k} i+$ $d_{k} a+e_{k} i a\left(b_{k}, c_{k}, d_{k}, e_{k} \in \mathbf{Z}\right)$, we have

$$
\begin{aligned}
&\left(\lambda_{1}, \lambda_{2}\right) \in \operatorname{Ker} \delta_{2}^{\#} \Leftrightarrow\left\{\begin{array}{l}
i \lambda_{1}+\lambda_{1} i=0, \\
a \lambda_{1}+\lambda_{1} a-\lambda_{1}+i \lambda_{2}+\lambda_{2} i=0, \\
a \lambda_{2}+\lambda_{2} a-\lambda_{2}=0
\end{array}\right. \\
& \Leftrightarrow\left\{\begin{array}{l}
-\left(2 c_{1}+d_{1}+e_{1}\right)+\left(2 b_{1}+d_{1}-e_{1}\right) i=0, \\
-\left(b_{1}+c_{1}+2 d_{1}+2 c_{2}+d_{2}+e_{2}\right) \\
+\left(2 b_{2}+d_{2}-e_{2}\right) i+\left(2 b_{1}+d_{1}-e_{1}\right) a=0, \\
-\left(b_{2}+c_{2}+2 d_{2}\right)+\left(2 b_{2}+d_{2}-e_{2}\right) a=0
\end{array}\right. \\
& \Leftrightarrow\left\{\begin{array}{l}
2 c_{1}+d_{1}+e_{1}=0 \\
2 b_{1}+d_{1}-e_{1}=0 \\
b_{1}+c_{1}+2 d_{1}+2 c_{2}+d_{2}+e_{2}=0 \\
b_{2}+c_{2}+2 d_{2}=0 \\
2 b_{2}+d_{2}-e_{2}=0
\end{array}\right.
\end{aligned}
$$




$$
\Leftrightarrow\left\{\begin{array}{l}
b_{1}=-c_{1}-2 d_{2}, \\
d_{1}=2 d_{2}, \\
e_{1}=-2 c_{1}-2 d_{2}, \\
c_{2}=-b_{2}-2 d_{2}, \\
e_{2}=2 b_{2}+d_{2} .
\end{array}\right.
$$

If this is the case, then we have

$$
\left(\lambda_{1}, \lambda_{2}\right)=c_{1}(i-1-2 i a, 0)-d_{2}(2-2 a+2 i a, 2 i-a-i a)+b_{2}(0,1-i+2 i a) .
$$

Therefore we have that

$\operatorname{Ker} \delta_{2}^{\#}=\mathbf{Z}(i-1-2 i a, 0) \oplus \mathbf{Z}(2-2 a+2 i a, 2 i-a-i a) \oplus \mathbf{Z}(0, i-1-2 i a)$.

Since for $\lambda_{1}=b_{1}+c_{1} i+d_{1} a+e_{1} i a\left(b_{1}, c_{1}, d_{1}, e_{1} \in \mathbf{Z}\right)$,

$$
\begin{aligned}
\delta_{1}^{\#}\left(\lambda_{1}\right)= & \left(i \lambda_{1}-\lambda_{1} i, a \lambda_{1}-\lambda_{1} a\right) \\
= & d_{1}(1-i+2 i a, 0)+e_{1}(1+i-2 a, 2 i-a-i a)+c_{1}(0, i-1-2 i a) \\
= & \left(e_{1}-d_{1}\right)(i-1-2 i a, 0)+e_{1}(2-2 a+2 i a, 2 i-a-i a) \\
& +c_{1}(0, i-1-2 i a),
\end{aligned}
$$

it follows that $\operatorname{Ker} \delta_{2}^{\#}=\operatorname{Im} \delta_{1}^{\#}$ holds.

(iii) The case $n(\geq 2)$ even: We show $H H^{n}(\Lambda)=\operatorname{Ker} \delta_{n+1}^{\#} / \operatorname{Im} \delta_{n}^{\#}=(\mathbf{Z} / 2 \mathbf{Z}) l_{n}^{1}$. First we calculate $\operatorname{Ker} \delta_{n+1}^{\#}$. Let $\lambda_{k}=b_{k}+c_{k} i+d_{k} a+e_{k} i a\left(b_{k}, c_{k}, d_{k}, e_{k} \in \mathbf{Z}\right)$. Since

$$
\begin{aligned}
&\left(\lambda_{1}, \lambda_{2}, \ldots, \lambda_{n+1}\right) \in \operatorname{Ker} \delta_{n+1}^{\#} \\
& \Leftrightarrow\left\{\begin{array}{l}
i \lambda_{1}-\lambda_{1} i=0, \\
a \lambda_{k}-\lambda_{k} a+i \lambda_{k+1}-\lambda_{k+1} i=0 \quad(k=1,2, \ldots, n), \\
a \lambda_{n+1}-\lambda_{n+1} a=0
\end{array}\right. \\
& \Leftrightarrow\left\{\begin{array}{l}
\left(d_{1}+e_{1}\right)+\left(e_{1}-d_{1}\right) i-2 e_{1} a+2 d_{1} i a=0, \\
\left(-c_{k}+d_{k+1}+e_{k+1}\right)+\left(c_{k}+2 e_{k}-d_{k+1}+e_{k+1}\right) i \\
-\left(e_{k}+2 e_{k+1}\right) a-\left(2 c_{k}+e_{k}-2 d_{k+1}\right) i a=0 \quad(k=1,2, \ldots, n), \\
-c_{n+1}+\left(c_{n+1}+2 e_{n+1}\right) i-e_{n+1} a-\left(2 c_{n+1}+e_{n+1}\right) i a=0
\end{array}\right. \\
& \Leftrightarrow\left\{\begin{array}{l}
d_{1}=0, \\
e_{k}=0 \\
c_{k}=d_{k+1} \quad(k=1,2, \ldots, n), \\
c_{n+1}=0
\end{array}\right. \\
& \Leftrightarrow\left(\begin{array}{l}
\left.\lambda_{1}, \lambda_{2}, \ldots, \lambda_{n+1}\right)=\sum_{k=1}^{n+1} b_{k} l_{n}^{k}+\sum_{k=1}^{n} c_{k}\left(i l_{n}^{k}+a l_{n}^{k+1}\right),
\end{array}\right.
\end{aligned}
$$


we have

$$
\begin{aligned}
\operatorname{Ker} \delta_{n+1}^{\#} & =\bigoplus_{k=1}^{n+1} \mathbf{Z} \imath_{n}^{k} \oplus \bigoplus_{k=1}^{n} \mathbf{Z}\left(i l_{n}^{k}+a l_{n}^{k+1}\right) \\
& =\bigoplus_{k=1}^{n+1} \mathbf{Z} \imath_{n}^{k} \oplus \bigoplus_{k=1}^{n} \mathbf{Z}\left((i+1) l_{n}^{k}+a l_{n}^{k+1}\right) .
\end{aligned}
$$

Next we prove

$$
\operatorname{Im} \delta_{n}^{\#}=\mathbf{Z}\left(2 \imath_{n}^{1}+l_{n}^{2}\right) \oplus \bigoplus_{k=2}^{n+1} \mathbf{Z} l_{n}^{k} \oplus \bigoplus_{k=1}^{n} \mathbf{Z}\left((i+1) l_{n}^{k}+a l_{n}^{k+1}\right) .
$$

For $\lambda_{k}=b_{k}+c_{k} i+d_{k} a+e_{k} i a\left(b_{k}, c_{k}, d_{k}, e_{k} \in \mathbf{Z}\right)$, we have

$$
\begin{aligned}
& \delta_{n}^{\#}\left(\lambda_{1}, \lambda_{2}, \ldots, \lambda_{n}\right)=\sum_{k=1}^{n} b_{k}\left(2 i l_{n}^{k}+(2 a-1) l_{n}^{k+1}\right)-\sum_{k=1}^{n} c_{k}\left(2 l_{n}^{k}+l_{n}^{k+1}\right) \\
& +\sum_{k=1}^{n} d_{k}\left((i-1) l_{n}^{k}+(a-2) l_{n}^{k+1}\right)-\sum_{k=1}^{n} e_{k}\left((i+1) l_{n}^{k}+a l_{n}^{k+1}\right) \\
& =\sum_{k=1}^{n}\left(2 b_{k}+d_{k}-e_{k}\right)\left((i+1) l_{n}^{k}+a l_{n}^{k+1}\right) \\
& -\sum_{k=1}^{n}\left(b_{k}+c_{k}+d_{k}\right)\left(2 l_{n}^{k}+l_{n}^{k+1}\right)-\sum_{k=1}^{n} d_{k} l_{n}^{k+1} \\
& =\sum_{k=1}^{n}\left(2 b_{k}+d_{k}-e_{k}\right)\left((i+1) l_{n}^{k}+a l_{n}^{k+1}\right) \\
& -\left(b_{1}+c_{1}+d_{1}\right)\left(2 l_{n}^{1}+l_{n}^{2}\right)-\left(d_{1}+2\left(b_{2}+c_{2}+d_{2}\right)\right) l_{n}^{2} \\
& -\sum_{k=2}^{n-1}\left(b_{k}+c_{k}+2\left(d_{k}+b_{k+1}+c_{k+1}+d_{k+1}\right)\right) l_{n}^{k+1} \\
& -\left(b_{n}+c_{n}+2 d_{n}\right) l_{n}^{n+1} .
\end{aligned}
$$

Then $\delta_{n}^{\#}\left(\lambda_{1}, \lambda_{2}, \ldots, \lambda_{n}\right)$ is an element of the right-hand side of (3.2). Conversely, by choosing $e_{k}(1 \leq k \leq n), b_{1}, d_{1}, b_{k}(2 \leq k \leq n)$ properly, $\delta_{n}^{\#}\left(\lambda_{1}, \lambda_{2}, \ldots, \lambda_{n}\right)$ is to be any element of the right-hand side of (3.2). Therefore we have

$$
\operatorname{Im} \delta_{n}^{\#}=2 \mathbf{Z} \imath_{n}^{1} \oplus \bigoplus_{k=2}^{n+1} \mathbf{Z}_{n}^{k} \oplus \bigoplus_{k=1}^{n} \mathbf{Z}\left((i+1) l_{n}^{k}+a l_{n}^{k+1}\right) .
$$

Hence by (3.1) and (3.3) we have the results. 


\section{Hochschild Cohomology Ring of $\Lambda$}

In this section, we determine the ring structure of the Hochschild cohomology ring $H H^{*}(\Lambda)$.

Recall the Yoneda product in $H H^{*}(\Lambda)$. Let $\alpha \in H H^{n}(\Lambda)$ and $\beta \in H H^{m}(\Lambda)$, where $\alpha$ and $\beta$ are represented by cocycles $f_{\alpha}: Y_{n} \rightarrow \Lambda$ and $f_{\beta}: Y_{m} \rightarrow \Lambda$, respectively. There exists the commutative diagram of $\Lambda^{\mathrm{e}}$-modules:

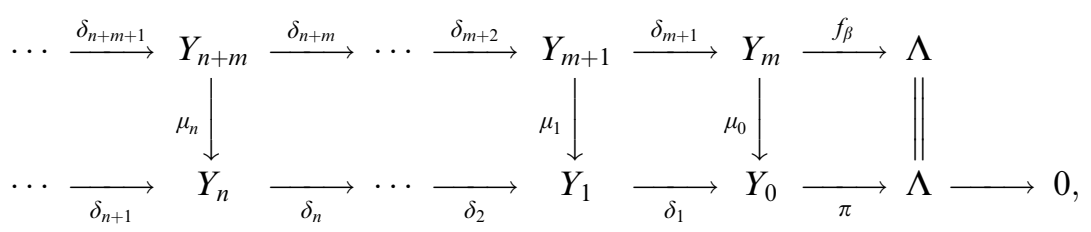

where $\mu_{l}(0 \leq l \leq n)$ are liftings of $f_{\beta}$. We define the product $\alpha \cdot \beta \in H H^{n+m}(\Lambda)$ by the cohomology class of $f_{\alpha} \mu_{n}$. This product is independent of the choice of representatives $f_{\alpha}$ and $f_{\beta}$, and liftings $\mu_{l}(0 \leq l \leq n)$.

Let $\alpha=l_{2}^{1}\left(\in H H^{2}(\Lambda)\right)$. Then $\alpha$ is represented by the $\Lambda^{\mathrm{e}}$-homomorphism $f_{\alpha}: Y_{2} \rightarrow \Lambda$ given by $f_{\alpha}\left(c_{2}^{1}\right)=1, f_{\alpha}\left(c_{2}^{2}\right)=f_{\alpha}\left(c_{2}^{3}\right)=0$. Then the following lemma holds.

Lemma 4.1. A lifting $\mu_{n}: Y_{n+2} \rightarrow Y_{n}$ of $f_{\alpha}$ is given by $\mu_{n}\left(c_{n+2}^{k}\right)=c_{n}^{k}$ for $n \geq 0$.

Proof. Clearly $\pi \cdot \mu_{0}=f_{\alpha}$ holds. If $n \geq 1$ is odd, we have

$$
\begin{aligned}
\mu_{n-1} \delta_{n+2}\left(c_{n+2}^{k}\right) & =\mu_{n-1}\left(i c_{n+1}^{k}-c_{n+1}^{k} i+a c_{n+1}^{k-1}-c_{n+1}^{k-1} a\right) \\
& =i c_{n-1}^{k}-c_{n-1}^{k} i+a c_{n-1}^{k-1}-c_{n-1}^{k-1} a \\
& =\delta_{n}\left(c_{n}^{k}\right)=\delta_{n} \mu_{n}\left(c_{n+2}^{k}\right) .
\end{aligned}
$$

The case $n \geq 2$ even is similar.

Let $\beta=l_{2 n}^{1} \in H H^{2 n}(\Lambda)$ for $n \geq 1$. Then $\beta$ is represented by the $\Lambda^{\mathrm{e}}$ homomorphism $f_{\beta}: Y_{2 n} \rightarrow \Lambda ; \quad c_{2 n}^{k} \mapsto l_{0}^{k}$. Since $f_{\beta} \cdot \mu_{2 n}\left(c_{2 n+2}^{k}\right)=f_{\beta}\left(c_{2 n}^{k}\right)=l_{0}^{k}$, it follows that $\alpha \beta=l_{2 n+2}^{1} \in H H^{2 n+2}(\Lambda)$ holds. Therefore we have $\alpha^{n}=l_{2 n}^{1} \in$ $H H^{2 n}(\Lambda)$ for $n \geq 1$, and the following theorem holds.

THEOREM 4.2. The Hochschild cohomology ring $H H^{*}(\Lambda)$ is isomorphic to $\mathbf{Z}[\alpha] /(2 \alpha)$, where $\operatorname{deg} \alpha=2$. 
REMARK. Let $\Gamma=\mathbf{Z} \oplus \mathbf{Z} i \oplus \mathbf{Z} j \oplus \mathbf{Z} i j$ be the ordinary quaternion $\mathbf{Z}$-algebra. The multiplicative structure of the Hochschild cohomology $\operatorname{ring} H H^{*}(\Gamma)$ is known in [6], [3]:

$$
H H^{*}(\Gamma) \simeq \mathbf{Z}[X, Y, Z] /\left(2 X, 2 Y, 2 Z, X^{2}+Y^{2}+Z^{2}\right),
$$

where $\operatorname{deg} X=\operatorname{deg} Y=\operatorname{deg} Z=1 . H H^{*}(\Gamma)$ is not a periodic cohomology. It is known that $\Gamma$ is not a maximal order of the ordinary quaternion $\mathbf{Q}$ algebra $A$. In general, there does not exist a ring homomorphism between two Hochschild cohomology rings except special cases. However, by Theorem 4.2, we get an injective ring homomorphism $H H^{*}(\Lambda) \rightarrow H H^{*}(\Gamma)$ given by $\alpha \mapsto X^{2}$.

\title{
References
}

[ 1 ] Bobovich, F. R., Cohomologies of maximal orders of simple central algebras, Math. Notes 6 (1969), 589-592; Engl. transl., Mat. Zametki 6 (1969), 225-231.

[2] Gerstenhaber, M., The cohomology structure of an associative ring, Ann. of Math. 78 (1963), 267-288.

[ 3 ] Hayami, T., Hochschild cohomology ring of an order of a simple component of the rational group ring of the generalized quaternion group, Comm. Algebra 36 (2008), 2785-2803.

[4] Hayami, T. and Sanada, K., Cohomology ring of the generalized quaternion group with coefficients in an order, Comm. Algebra 30 (2002), 3611-3628.

[ 5 ] Rickard, J., Derived equivalences as derived functors, J. London Math. Soc. (2) 43 (1991), $37-48$.

[6] Sanada, K., On the Hochschild cohomology of crossed products, Comm. Algebra 21 (1993), 2727-2748

[ 7 ] Sanada, K., Remarks on cohomology rings of the quaternion group and the quaternion algebra, SUT J. Math. 31 (1995), 85-92.

\author{
Department of Mathematics \\ Hokkai-Gakuen University \\ 4-1-40, Asahi-machi, Toyohira-ku \\ Sapporo 062-8605, Japan \\ E-mail: hayami@ma.kagu.tus.ac.jp
}

